\title{
INVARIANT SUBSPACE LATTICES OF LAMBERT'S WEIGHTED SHIFTS
}

\author{
B. S. YADAV and S. CHATTERJEE \\ (Received 25 January 1980; revised 2 December 1980) \\ Communicated by J. B. Miller
}

\begin{abstract}
Let $B(H)$ be the Banach algebra of all (bounded linear) operators on an infinite-dimensional separable complex Hilbert space $H$ and let $\left\{a_{m}\right\}_{m=0}^{\infty}$ be a bounded sequence of positive real numbers. For a given injective operator $A$ in $B(H)$ and a non-zero vector $f$ in $H$, we put $w_{m}=$ $a_{m}\left\|A^{m+1} f\right\| /\left\|A^{m} f\right\|, m=0,1,2, \ldots$. We define a weighted shift $T_{w}$ with the weight sequence $w=\left\{w_{m}\right\}_{m=0}^{\infty}$ on the Hilbert space $1^{2}$ of all square-summable complex sequences $x=\left\{x_{0}, x_{1}, x_{2}, \ldots\right\}$ by $T_{w}(x)=\left\{0, w_{0} x_{0}, w_{1} x_{1}, w_{2} x_{2}, \ldots\right\}$. The main object of this paper is to characterize the invariant subspace lattice of $T_{w}$ under various nice conditions on the operator $A$ and the sequence $\left\{a_{m}\right\}_{m=0}^{\infty}$.

1980 Mathematics subject classification (Amer. Math. Soc.): primary 47 A 15, 47 B 99; secondary 47 C 05 .
\end{abstract}

\section{Introduction}

Let $H$ be an infinite-dimensional separable complex Hilbert space and $B(H)$ the algebra of all (bounded linear) operators from $H$ into $H$. If $H$ is $1^{2}$, that is, the Hilbert space of all square-summable complex sequences $x=\left\{x_{0}, x_{1}, x_{2}, \ldots\right\}$ with the norm

$$
\|x\|=\left(\sum_{m=0}^{\infty}\left|x_{m}\right|^{2}\right)^{1 / 2},
$$

and if $\alpha=\left\{\alpha_{m}\right\}_{m=0}^{\infty}$ is a bounded sequence of non-zero complex numbers, then the operator $T_{\alpha}$ on $1^{2}$ defined by

$$
T_{\alpha}\left\{x_{0}, x_{1}, x_{2}, \ldots\right\}=\left\{0, \alpha_{0} x_{0}, \alpha_{1} x_{1}, \alpha_{2} x_{2}, \ldots\right\}
$$

(c) Copyright Australian Mathematical Society 1982 
is called a (unilateral forward) weighted shift on $1^{2}$ with the weight sequence $\alpha=\left\{\alpha_{m}\right\}_{m=0}^{\infty}$. We may, and shall assume, without any loss of generality that the weights $\alpha_{m}$ are positive real numbers [3, Problem 2]. The invariant subspaces of this class of operators have been extensively studied by many authors; see, for example, Donoghue [1], Nikolskiĩ [7], [8], [9], Kelley [5], Nordgren [10], Harrison [4] and Shields [13].

By an invariant subspace $M$ of $T$ we shall mean a closed linear manifold of $1^{2}$ such that $T M \subset M$. By Lat $T$ we shall denote the lattice of invariant subspaces of $T$. The object of this paper is to characterize the lattice of a rather specialised class of weighted shifts. Such weighted shifts have recently been studied for their subnormality by Lambert [6].

Let $A$ be an injective operator on $H$ and suppose that $\left\{a_{m}\right\}_{m=0}^{\infty}$ is a bounded sequence of positive real numbers. For each non-zero vector $f$ in $H$, let $T_{w}$ be the weighted shift on $1^{2}$ with the weight sequence $w=\left\{w_{m}\right\}_{m=0}^{\infty}$, where

$$
w_{m}=a_{m} \frac{\left\|A^{m+1} f\right\|}{\left\|A^{m} f\right\|} .
$$

A vector $x$ in $1^{2}$ is called a cyclic vector of $T_{w}$ if

$$
1^{2}=\bigvee_{n=0}^{\infty}\left\{T_{w}^{n} x\right\}
$$

the subspace spanned by $x, T_{w} x, T_{w}^{2} x, \ldots$

A sequence $\left\{a_{m}\right\}_{m=0}^{\infty}$ is said to be of bounded variation if

$$
\sum_{m=0}^{\infty}\left|a_{m}-a_{m+1}\right|<\infty
$$

It is easy to see that if $\left\{a_{m}\right\}_{m=0}^{\infty}$ is monotonically decreasing, then it is of bounded variation, but the converse is not true. We shall say that $\left\{a_{m}\right\}_{m=0}^{\infty}$ is in the class $B V(*)$ if it is of bounded variation and satisfies the condition:

$$
\Delta=\sup _{m \geqslant 2, n} \sum_{k=0}^{\infty}\left(\frac{a_{k+m} \cdots a_{k+n}}{a_{m} a_{m+1} \cdots a_{n}}\right)^{2}<\infty .
$$

An operator $A$ in $B(H)$ is power-bounded if

$$
\left\|A^{n}\right\| \leqslant \delta
$$

for all $n=1,2,3, \ldots$, where $\delta$ is a constant. We first prove 
LEMMA 2.1. Let $A$ be power-bounded and such that for every non-zero vector $f$ in $H, A^{n} f \nrightarrow 0$ as $n \rightarrow \infty$. If the sequence $\left\{a_{m}\right\}_{m=0}^{\infty}$ is in $B V(*)$, then any vector $x=\left\{x_{m}\right\}_{m=0}^{\infty}$ in $1^{2}$ with $x_{0} \neq 0$ is a cyclic vector of $T_{w}$.

Proof. We first observe that

$$
\inf _{n \geq 0}\left\|A^{n} f\right\|=\mu(f)>0 \quad \text { for all } f \neq 0 .
$$

In fact $\mu(f)=0$ implies that there exists, for every $\varepsilon>0$, an $n_{0}=n_{0}(f, \varepsilon)$ such that $\left\|A^{n_{0}} f\right\|<\varepsilon / \delta$; and hence

$$
\left\|A^{n} f\right\|=\left\|A^{n-n_{0}} A^{n_{0}} f\right\| \leqslant \delta\left\|A^{n_{0}} f\right\|<\varepsilon
$$

for $n \geqslant n_{0}$. This contradicts our hypothesis that $A^{n} f \nrightarrow 0$.

Let $\left\{e_{m}\right\}_{m=0}^{\infty}$ be the standard orthonormal basis of $1^{2}$. As

$$
T_{w}^{n} x=\{\underbrace{0,0, \ldots, 0}_{n}, x_{0} w_{0} w_{1} \cdots w_{n-1}, x_{1} w_{1} w_{2} \cdots w_{n}, \ldots\},
$$

we have

$$
\begin{aligned}
\left\|\frac{T_{w}^{n} x}{x_{0} w_{0} w_{1} \cdots w_{n-1}}-e_{n}\right\|^{2}=\sum_{m=0}^{\infty}\left(\frac{w_{m+1} \cdots w_{m+n}}{w_{0} w_{1} \cdots w_{n-1}}\right)^{2}\left|\frac{x_{m+1}}{x_{0}}\right|^{2} \\
\quad=\left.\left.\sum_{m=0}^{\infty}\left(\frac{a_{m+1} \cdots a_{m+n}}{a_{0} a_{1} \cdots a_{n-1}}\right)^{2} \frac{\left\|A^{m+n+1} f\right\|^{2}\|f\|^{2}}{\left\|A^{m+1} f\right\|^{2}\left\|A^{n} f\right\|^{2}}\right|^{\frac{x_{m+1}}{x_{0}}}\right|^{2} \quad(\text { by (1)) } \\
\quad \leqslant \frac{\left\|A^{n}\right\|^{2}\|f\|^{2}}{\left\|A^{n} f\right\|^{2}} \sum_{m=0}^{\infty}\left(\frac{a_{m+1} \cdots a_{m+n}}{a_{0} a_{1} \cdots a_{n-1}}\right)^{2}\left|\frac{x_{m+1}}{x_{0}}\right|^{2} \\
\leqslant \frac{\delta^{2}\|f\|^{2}\|x\|^{2}}{(\mu(f))^{2}\left|x_{0}\right|^{2}} \sum_{m=0}^{\infty}\left(\frac{a_{m+1} \cdots a_{m+n}}{a_{0} a_{1} \cdots a_{n-1}}\right)^{2} \quad(\text { by (2) and (3)) } \\
=\frac{\delta^{2}\|f\|^{2}\|x\|^{2} a_{n}^{2}}{(\mu(f))^{2}\left|x_{0}\right|^{2}\left(a_{0} a_{1}\right)^{2} \sum_{m=0}^{\infty}\left(\frac{a_{m+2} \cdots a_{m+n}}{a_{2} \cdots a_{n}}\right)^{2} a_{m+1}^{2}} \\
=C a_{n}^{2} \sum_{m=0}^{\infty} \sum_{k=0}^{m}\left(\frac{a_{k+2} \cdots a_{k+n}}{a_{2} \cdots a_{n}}\right)^{2}\left(a_{m+1}^{2}-a_{m+2}^{2}\right) \\
\leqslant C \Delta a_{n}^{2} \sum_{m=0}^{\infty}\left(a_{m+1}^{2}-a_{m+2}^{2}\right) \quad(\text { by }(*)) \\
\leqslant 2 a C \Delta a_{n}^{2} \sum_{m=0}^{\infty}\left|a_{m+1}-a_{m+2}\right| \leqslant C a_{n}^{2},
\end{aligned}
$$


where $a=\sup _{m}\left\{a_{m}\right\}$ and $C$ denotes a constant not necessarily the same everywhere.

Since $\left\{e_{n}\right\}_{n=0}^{\infty}$ is an orthonormal basis in $\mathbf{1}^{2}$, and by $(*) \sum_{n=0}^{\infty} a_{n}^{2}<\infty$, it follows by the Paley-Wiener theorem [12, page 208] that the system

$$
\left\{\frac{T_{w}^{n} x}{x_{0} w_{0} w_{1} \cdots w_{n-1}}\right\}_{n=0}^{\infty}
$$

is a Riesz basis in $1^{2}$, whence we conclude that

$$
\bigvee_{n=0}^{\infty}\left\{T_{w}^{n} x\right\}=1^{2}
$$

An operator $A$ in $B(H)$ is said to belong to the class $C_{1}$. if it is a contraction (that is $\|A\| \leqslant 1$ ) and $A^{n} f \nrightarrow 0$ for all $f \neq 0$. The class $C_{1}$. plays an important role in the study of general contractions [14, page 72]. The following special case of Lemma 2.1 is worth mention:

COROLlaRY 2.2. Lemma 2.1 holds if $\left\{a_{m}\right\}_{m=0}^{\infty}$ is a monotonically decreasing square-summable sequence and $A \in C_{1}$. .

Define

$$
M_{k}=\left\{x=\left\{x_{m}\right\}_{m=0}^{\infty} \in 1^{2} ; x_{m}=0, m<k\right\}, \quad k=1,2, \ldots .
$$

It is obvious that $M_{k} \supset M_{k+1}$ and $M_{k} \in$ Lat $T_{w}$ for all $k=1,2, \ldots$ We show that the $M_{k}$ are the only non-trivial invariant subspaces of $T_{w}$ when $A$ and $\left\{a_{m}\right\}_{m=0}^{\infty}$ satisfy the hypothesis of Lemma 2.1 .

THEOREM 2.3. Let $A$ be power-bounded and such that for every non-zero vector $f$ in $H, A^{n} f \nrightarrow 0$ as $n \rightarrow \infty$. If the sequence $\left\{a_{m}\right\}_{m=0}^{\infty}$ is in $B V(*)$, then Lat $T_{w}$ is order-isomorphic to $1+{ }^{*} \omega$, where ${ }^{*} \omega$ denotes the order-type of the negative integers [11, page 26].

Proof. Let $M$ be a non-trivial invariant subspace of $T_{w}$. If $x=\left\{x_{m}\right\}_{m=0}^{\infty}$ is any vector in $M$, then, in view of Lemma 2.1, $x_{0}=0$. Suppose that $k$ is the least positive integer such that $x_{k} \neq 0$. We first show that

$$
\bigvee_{n=0}^{\infty}\left\{T_{w}^{n} x\right\}=M_{k} \text {. }
$$


Recalling that $\left\{e_{m}\right\}_{m=k}^{\infty}$ is an orthonormal basis of $M_{k}$ and following the proof of Lemma 2.1, we have

$$
\begin{aligned}
& \left\|\frac{T_{w}^{n} x}{x_{k} w_{k} w_{k+1} \cdots w_{k+n-1}}-e_{n+k}\right\|^{2} \\
& \quad=\sum_{m=0}^{\infty}\left(\frac{a_{m+k+1} \cdots a_{m+k+n}}{a_{k} a_{k+1} \cdots a_{k+n-1}}\right)^{2} \frac{\left\|A^{k+m+n+1} f\right\|^{2}\left\|A^{k} f\right\|^{2}}{\left\|A^{k+m+1} f\right\|^{2}\left\|A^{k+n} f\right\|^{2}}\left|\frac{x_{m+k+1}}{x_{k}}\right|^{2} \\
& \leqslant \\
& \leqslant \frac{\left\|A^{n}\right\|^{2}\left\|A^{k} f\right\|^{2}}{\left\|A^{k+n} f\right\|^{2}} \sum_{m=0}^{\infty}\left(\frac{a_{m+k+1} \cdots a_{m+k+n}}{a_{k} a_{k+1} \cdots a_{k+n-1}}\right)^{2}\left|\frac{x_{m+k+1}}{x_{k}}\right|^{2} \\
& \leqslant \frac{\delta^{4}\|f\|^{2}\|x\|^{2} a_{k+n}^{2}}{(\mu(f))^{2}\left|x_{k}\right|^{2}\left(a_{k} a_{k+1}\right)^{2}} \sum_{m=0}^{\infty}\left(\frac{a_{m+k+2} \cdots a_{m+k+n}}{a_{k+2} \cdots a_{k+n}}\right)^{2} a_{m+k+1}^{2} \\
& \leqslant C a_{k+n}^{2} .
\end{aligned}
$$

It is now immediate by the Paley-Wiener theorem that $\bigvee_{n=0}^{\infty}\left\{T_{w}^{n} x\right\}=M_{k}$.

Since the span of any number of $M_{k}$ is again an $M_{k}$, we conclude that $M=M_{k}$. Consequently, we have

$$
\text { Lat } T_{w}=\left\{\{0\}, \ldots, M_{3}, M_{2}, M_{1}, 1^{2}\right\}
$$

and thus Lat $T_{w}$ is order-isomorphic to $1+{ }^{*} \omega$.

Corollary 2.4. Let $A$ be invertible with both $A$ and $A^{-1}$ power-bounded. If the sequence $\left\{a_{m}\right\}_{m=0}^{\infty}$ is in $B V(*)$, then Lat $T_{w}$ is order-isomorphic to $1+{ }^{*} \omega$.

Proof. If $\left\|A^{n}\right\| \leqslant \delta, n=0, \pm 1, \pm 2, \ldots$, then $\left\|A^{n} f\right\| \geqslant(1 / \delta)\|f\|$, so $f \neq 0 \Rightarrow$ $A^{n} f \nrightarrow 0$.

We now consider the Hilbert space $1^{2}\left(\mathbf{C}^{k}\right), k \geqslant 1$ of norm-square-summable sequences of vectors of the $k$-dimensional unitary spaces $\mathbf{C}^{k}$. Thus $1^{2}\left(\mathbf{C}^{k}\right)$ consists of sequences

$$
x=\left\{x_{m}\right\}_{m=0}^{\infty}, \quad x_{m} \in \mathbf{C}^{k}
$$

such that $\sum_{m=0}^{\infty}\left\|x_{m}\right\|_{*}^{2}<\infty$, where $\left\|x_{m}\right\|_{*}$ is the norm of $x_{m}$ in $\mathbf{C}^{k}$ and

$$
\|x\|=\left(\sum_{m=0}^{\infty}\left\|x_{m}\right\|_{*}^{2}\right)^{1 / 2} .
$$


Although we have not been able to prove the analogues of Theorem 2.3 and Corollary 2.4 for the Hilbert space $1^{2}\left(\mathbf{C}^{k}\right)$, we shall, however, show that Lemma 2.1 has an interesting extension in this case.

We shall say that a non-empty subset $S$ of $1^{2}\left(\mathbf{C}^{k}\right)$ is a cyclic set of an operator $T$ on $1^{2}\left(\mathbf{C}^{k}\right)$ if

$$
\bigvee_{n=0}^{\infty}\left\{T^{n} x: x \in S\right\}=1^{2}\left(C^{k}\right)
$$

THEOREM 3.1. Let $A$ be power-bounded and such that for every non-zero vector $f$ in $H, A^{n} f \nrightarrow 0$ as $n \rightarrow \infty$. If the sequence $\left\{a_{m}\right\}_{m=0}^{\infty}$ is in $B V(*)$, then any set of $k$-vectors in $1^{2}\left(\mathbf{C}^{k}\right)$ such that their first coordinates form a basis of $\mathbf{C}^{k}$ is a cyclic set of the weighted shift $T_{n}$ on $1^{2}\left(\mathbf{C}^{k}\right)$.

PROOF. Let $x^{(i)}=\left\{x_{m}^{(i)}\right\}_{m=0}^{\infty}, i=1,2, \ldots, k$, be $k$ elements of $1^{2}\left(\mathbf{C}^{k}\right)$ such that $\left\{x_{0}^{(1)}, x_{0}^{(2)}, \ldots, x_{0}^{(k)}\right\}$ is a basis in $\mathbf{C}^{k}$. We assume, without any loss of generality, that $\left\{x_{0}^{(1)}, x_{0}^{(2)}, \ldots, x_{0}^{(k)}\right\}$ is an orthonormal basis in $\mathbf{C}^{k}$. Then

$$
T_{n}^{n} x^{(i)}=\{\underbrace{0,0, \ldots, 0}_{n}, w_{n-1} \cdots w_{1} w_{0} x_{0}^{(i)}, w_{n} \cdots w_{2} w_{1} x_{1}^{(i)}, \ldots\} .
$$

If $e_{n}(z), z \in \mathbf{C}^{k}$, denotes the element of $1^{2}\left(\mathbf{C}^{k}\right)$ having $z$ in the $n$th place and 0 elsewhere, we have

$$
\left\|\frac{T_{w}^{n} x^{(i)}}{w_{0} w_{1} \cdots w_{n-1}}-e_{n}\left(x_{0}^{(i)}\right)\right\|^{2}=\sum_{m=0}^{\infty}\left(\frac{w_{m+1} \cdots w_{m+n}}{w_{0} w_{1} \cdots w_{n-1}}\right)^{2}\left\|x_{m+1}^{(i)}\right\|_{*}^{2} \leqslant C a_{n}^{2}\left\|x^{(i)}\right\|^{2} .
$$

Since $\left\{e_{n}\left(x_{0}^{(i)}\right)\right\}_{n \geqslant 0,1 \leqslant i \leqslant k}$ is an orthonormal basis in $1^{2}\left(\mathbf{C}^{k}\right)$ and by $(*)$

$$
\sum_{\substack{n \geq 0 \\ 1 \leqslant i \leqslant k}} a_{n}^{2}\left\|x^{(i)}\right\|^{2}<\infty
$$

it follows that the system

$$
\left\{\frac{T_{w}^{n} x^{(i)}}{w_{0} w_{1} \cdots w_{n-1}}\right\}_{n \geqslant 0,1 \leqslant i \leqslant k}
$$

is a Riesz basis in $1^{2}\left(\mathbf{C}^{k}\right)$ and consequently $\left\{x^{(i)}\right\}_{i=1}^{k}$ is a cyclic set of $T_{n}$.

Corollary 3.2. Let $A$ be invertible with both $A$ and $A^{-1}$ power-bounded and suppose that $\left\{a_{m}\right\}_{m=0}^{\infty}$ is in $B V(*)$. Then any set of $k$-vectors in $1^{2}\left(C^{k}\right)$ such that their first coordinates form a basis of $\mathbf{C}^{k}$ is a cyclic set of $T_{n}$.

A strictly cyclic operator algebra $\mathscr{Q}$ on $H$ is a uniformly closed subalgebra of $B(H)$ such that $Q f_{0}=H$ for some vector $f_{0}$ in $H$. In this case $f_{0}$ is called a strictly 
cyclic vector for $Q$. Moreover, if $A f_{0}=0, A \in \mathbb{Q}$ implies that $A=0$, we say that $f_{0}$ is a separating vector for $\mathcal{Q}$. The following lemma is due to Embry [2]:

LEMMA 3.3. Let $f_{0}$ be a strictly cyclic separating vector for $\mathcal{Q}$. Then there exists a constant $C$ such that

$$
\|A\| \leqslant C\left\|A f_{0}\right\|
$$

for every $A$ in $\&$.

THEOREM 3.4. Let $Q$ be a strictly cyclic operator algebra with a strictly cyclic separting vector $f_{0}$, and let $A \in \mathbb{Q}$. If the sequence $\left\{a_{m}\right\}_{m=0}^{\infty}$ is in $B V(*)$, then any set of $k$-vectors in $1^{2}\left(\mathbf{C}^{k}\right)$ such that their first coordinates form a basis of $\mathbf{C}^{k}$ is a cyclic set of $T_{w}$, where the weight sequence $w=\left\{w_{m}\right\}_{m=0}^{\infty}$ is defined by $w_{m}=$ $a_{m}\left\|A^{m+1} f_{0}\right\| /\left\|A^{m} f_{0}\right\|$.

Proof. Following the proof of Theorem 3.1, it suffices to observe that

$$
\begin{aligned}
& \left\|\frac{T_{w}^{n} x^{(i)}}{w_{0} w_{1} \cdots w_{n-1}}-e_{n}\left(x_{0}^{(i)}\right)\right\|^{2} \leqslant \frac{\left\|A^{n}\right\|^{2}\left\|f_{0}\right\|^{2}}{\left\|A^{n} f_{0}\right\|^{2}} \sum_{m=0}^{\infty}\left(\frac{a_{m+1} \cdots a_{m+n}}{a_{0} a_{1} \cdots a_{n-1}}\right)^{2}\left\|x_{m+1}^{(i)}\right\|_{*}^{2} \\
& \quad \leqslant C\left\|f_{0}\right\|^{2} \sum_{m=0}^{\infty}\left(\frac{a_{m+1} \cdots a_{m+n}}{a_{0} a_{1} \cdots a_{n-1}}\right)^{2}\left\|x_{m+1}^{(i)}\right\|_{*}^{2} \quad \text { (by Lemma 3.3) } \\
& \quad \leqslant C a_{n}^{2}\left\|x^{(i)}\right\|^{2} .
\end{aligned}
$$

The authors thank the referee, and also the editor, for their suggestions to rewrite the paper in the present form.

\section{References}

1. W. F. Donoghue, 'The lattice of invariant subspaces of a completely continuous quasi-nilpotent transformation', Pacific J. Math. 7 (1957), 1031-1035.

2. Mary R. Embry, 'Strictly cyclic operator algebras on a Banach space', Pacific J. Math. 45 (1973), 443-452.

3. P. R. Halmos, 'Ten problems in Hilbert space', Bull. Amer. Math. Soc. 78 (1970), 887-933.

4. K. J. Harrison, 'On the unicellularity of weighted shifts', J. Austral. Math. Soc. 12 (1971). 342-350.

5. R. L. Kelley, Weighted shifts on Hilbert space (Thesis, University of Michigan, Ann Arbor. Mich., 1966).

6. Alan Lambert, 'Subnormality and weighted shifts', J. London Math. Soc. (2) 14 (1976), 476-480.

7. N. K. Nikolskī, 'Invariant subspaces of certain completely continuous operators', Vestmik Leningrad. Univ. Math. (7) 20 (1965), 68-77 (Russian). 
8. N. K. Nikolskiī, 'Invariant subspaces of weighted shift operators', Math. USSR-Sb. 3 (1967), 159-176.

9. N. K. Nikolskii, 'Basiness and unicellularity of weighted shift operators', Functional Anal. Appl. $2(1968), 95-98$.

10. Eric A. Nordgren, 'Invariant subspaces of a direct sum of weighted shifts', Pacific J. Math. 27 (1968), 587-598.

11. William J. Pervin, Foundations of general topologv (Academic Press, New York, 1970).

12. F. Riesz and B. Sz.-Nagy, Functional analysis (Ungar, New York, 1955).

13. A. Shields, Weighted shift operators and analytic function theory (Mathematical Surveys, No. 13, edited by C. Pearcy, Amer. Math. Soc., Providence, R. I., 1974).

14. B. Sz.-Nagy and C. Foiaş, Harmonic analysis of operators on Hilbert space (Akadémiai Kiadó, Budapest, 1970).

15. A. Zygmund, Trigonometric series (Vol. I, 2nd ed., Cambridge Univ. Press, New York, 1959).

\section{Department of Mathematics}

University of Delhi

Delhi-1 10007

India 